\title{
Distinct roles of different fragments of PDCD4 in regulating the metastatic behavior of B16 melanoma cells
}

\author{
DI WANG, SHU GUO, SI-YUAN HAN, NAN XU, JIA-YAN GUO and QING SUN \\ Department of Plastic Surgery, The First Hospital of China Medical University, Shenyang, Liaoning 110001, P.R. China
}

Received December 12, 2012; Accepted January 29, 2013

DOI: 10.3892/ijo.2013.1841

\begin{abstract}
Melanoma is an aggressive cutaneous malignancy. In this study, we demonstrated that the levels of the programmed cell death 4 (PDCD4) protein and mRNA were lower in tumor tissues compared with normal tissues. In order to further investigate the effects of PDCD4 and its fragments in B16 melanoma cells, we established B16 clones with expression of different PDCD4 fragments. Intact PDCD4, PDCD4 $164-469$ and PDCD4 327 -440 expression, respectively, decreased proliferation and migration and increased apoptosis in B16 cells in vitro. We found that intact PDCD4, PDCD4 $164-469$ or PDCD4 $327-440$ can inhibit the activity of MMP-2 and the expression of CXCR4. However, PDCD4 $4164-275$ showed no effects on B16 cells. These results may prove helpful for the development of novel therapies for melanoma treatment.
\end{abstract}

\section{Introduction}

Melanoma is an aggressive cutaneous malignancy accounting for just $4 \%$ of skin cancers but resulting in $80 \%$ of all skin-cancer related deaths (1). Melanoma may be induced by continuous proliferation of the melanocytes and dysregulation of the epidermal melanin unit (2). Melanocyte growth is controlled by the surrounding keratinocytes by intercellular communication through cell-cell adhesion molecules, cell-matrix adhesion, and gap junctional intercellular communication (3). A previous study has confirmed that downregulation of programmed cell death 4 (PDCD4) leads to the downregulation of E-cadherin and urokinase-type plasminogen activator receptor, which leads to degradation of extracellular matrix and invasion (4).

$P D C D 4$ was first identified by differential display that is upregulated upon in apoptosis-induced murine cell lines (5). The human PDCD4 gene is localized in chromosome 10q24 (6). PDCD4 protein can suppress tumor promotion and progression to carcinomas in cultured cells and transgenic

Correspondence to: Dr Shu Guo, Department of Plastic Surgery, The First Hospital of China Medical University, Nanjing North Street 155, Heping District, Shenyang, Liaoning 110001, P.R. China E-mail: guos_cmu@163.com

Key words: PDCD4, melanoma, apoptosis, CXCR4, metastasis mice (7,8). As shown in Fig. 1, full length human PDCD4 contains an N-terminal putative RNA-binding domain (residues 1-140) and two tandem MA3 domains, designated as nMA3 (residues 164-275) and cMA3 (residues 327-440) (9). The two MA3 domains have very similar structure and eIF4A-binding surfaces (10). The molecular basis for PDCD4mediated suppression has been linked to its high affinity MA-3 domains $(11,12)$. NMR binding analysis has shown that both MA3 domains of PDCD4 interacted with eIF4A and prevented translation $(10,11)$. However, another study has demonstrated that the cMA3 domain alone is sufficient for the inhibition of RNA helicase and translation (13). PDCD4 expression is significantly downregulated in various human cancers such as lung cancer (14), hepatocellular carcinoma (15), breast carcinoma (16), colorectal cancer (17) and gastric cancer (18). PDCD4 can induce tumor cell to apoptosis, inhibit tumor angiogenesis and increase sensitivity of tumor cells to antitumor drugs or radiotherapy $(19,20)$. Recent studies showed the indirect relationship between PDCD4 and melanoma cells (21-23), however, to our knowledge, little is known yet about the individual role of cMA3 or nMA3 of PDCD4 in B16 melanoma cells.

In our study, we showed that cMA3 domain of PDCD4 caused profound suppression of cell proliferation in B16 melanoma cells. In contrast to the role of cMA3, our findings showed that the antitumor effects of nMA3 were weak relatively in B16 melanoma cells. The cMA3 domain of PDCD4 may serve as an effective drug independently.

\section{Materials and methods}

Cell lines and culture. The human melanoma cell line, B16, was obtained from the American Type Culture Collection (ATCC, Bethesda, MD). Cell lines were grown in Dulbecco's modified Eagle's medium (Hyclone, Logan, UT) supplemented with $10 \%$ fetal bovine serum and antibiotics $(100 \mathrm{U} / \mathrm{ml}$ penicillin and $100 \mu \mathrm{g} / \mathrm{ml}$ streptomycin) and maintained in a humidified incubator with $5 \% \mathrm{CO}_{2}$ at $37^{\circ} \mathrm{C}$.

Subjects. Total specimens of 21 patients with melanoma were obtained from the Department of Plastic Surgery, The First Affiliated Hospital of China Medical University (Jan. 2001 to Dec. 2010). None of the patients underwent radiotherapy or chemotherapy before operation. This study was in compliance with the Helsinki Declaration, all patients gave written 
informed consent for participation and the procedure was approved by Our University Ethics Committee.

Immunohistochemical staining. All tissues were routinely fixed in $10 \%$ buffered formalin and embedded in paraffin. Sections $(4 \mu \mathrm{m})$ were deparaffinized in xylene. Endogenous peroxidase was blocked with $3 \%$ hydrogen peroxide in deionized water for $20 \mathrm{~min}$. Antigen retrieval was carried out in citrate buffer $(10 \mathrm{mM}, \mathrm{pH} 6.0)$ for $30 \mathrm{~min}$ at $95^{\circ} \mathrm{C}$. Section were immunostained with polyclone antibody to PDCD4 (sc-27123; Santa Cruz Biotechnology, Santa Cruz, CA) at 1:200 dilution or CXCR4 (PA1237; Boster Biological Technology, Fremont, CA) at 1:50 dilution for $60 \mathrm{~min}$ at $37^{\circ} \mathrm{C}$, followed by biotinylated secondary antibody for $30 \mathrm{~min}$, subsequently reacted with by HRP for $30 \mathrm{~min}$. For visualization, hydrogen peroxide-activated diamino benzidine (DAB) was applied. Washes (50-min) in PBS were carried out between each step. Tissue sections were lightly counterstained with hematoxylin, dehydrated through graded alcohols, cleared with xylene and mounted in mounting medium. Normal tissue was used as a control. Sections treated without primary antibodies were used as negative controls.

SDS-PAGE and immunoblotting. Proteins (45 $\mu \mathrm{g}$ per lane) were separated by $10 \%$ SDS-polyacrylamide gel electrophoresis and transferred to PVDF membranes (Millipore Corp., Billerica, MA). Western blotting was performed using primary antibodies: PDCD4 (sc-27123; Santa Cruz Biotechnology), CXCR4 (PA1237; Boster Biological Technology) and $\beta$-actin (sc-47778; Santa Cruz Biotechnology). Incubation with antibodies was performed in $1.5 \%$ BSA in TBS, $0.1 \%$ Tween. Detection of the immune complexes was performed with the ECL western blotting detection system (Amersham Biosciences, Piscataway, NJ).

Construction of PDCD4 cDNA expression vector. For the preparation of truncated PDCD4 proteins, the relevant sequences were amplified from full-length PDCD4 (GenBank accession no. NM_014456.3) by PCR using primers that included designed restriction sites (Table I). PDCD4 and the truncated forms created in this study are presented in Fig. 1. cDNAs of the truncated PDCD4 forms were obtained by PCR, then digested with the relevant restriction enzymes and ligated into pcDNA3.1. Transfection of plasmids into B16 cells was performed using Lipofectamine ${ }^{\mathrm{TM}} 2000$ (Invitrogen, Carlsbad, $\mathrm{CA})$ according to the manufacturer's instructions.

Real-time PCR and RT-PCR. Total tissue and cellular RNA was isolated using TRIzol reagent (Invitrogen). cDNA was then synthesized from $1 \mu \mathrm{g}$ of total RNA using SuperScript II reverse transcriptase (Invitrogen) according to the manufacturer's protocol. The level of tissular PDCD 4 mRNA was quantitated by real-time PCR (ABI PRISM 7500) using power SYBR $^{\circledR}$ Green PCR Master Mix (Takara Dalian, Dalian, China) and specific primers for PDCD4 and GAPDH. The following primer sets were used: $P D C D 4$ sense, 5'-GTATGAT GTGGAGGAGGTGGAT-3'; antisense: 5'-CCCTCCAATGCT AAGGATACTG-3'; GAPDH sense, 5'-GAAGGTGAAGGTCG GAGT-3'; antisense:5'-CATGGGTGGAATCATATTGGAA-3'. The real-time PCR conditions were as follows: one cycle at $95^{\circ} \mathrm{C}$ for $10 \mathrm{~min}$ followed by 40 cycles at $95^{\circ} \mathrm{C}$ for $15 \mathrm{sec}$ and at $60^{\circ} \mathrm{C}$ for $1 \mathrm{~min}$. Each reaction was repeated independently three times in triplicate. Results for the quantity of mRNA were expressed as the number of copies of $P D C D 4$ mRNA per copy of GAPDH mRNA.

PCR amplification of cellular cDNA was performed in $15-\mu 1$ mixtures. The primers are summarized in Table I. The RT-PCR conditions were: one cycle at $94^{\circ} \mathrm{C}$ for $5 \mathrm{~min}$, followed by 35 cycles of $94^{\circ} \mathrm{C}$ for $30 \mathrm{sec}, 72^{\circ} \mathrm{C}$ for $45 \mathrm{sec}, 58^{\circ} \mathrm{C}$ for $45 \mathrm{sec}$ and final extension at $72^{\circ} \mathrm{C}$ for $10 \mathrm{~min}$. Finally, products were resolved by $1 \%$ agarose gel electrophoresis and visualized by ethidium bromide staining and a UV imaging system (UVP, Upland, CA).

Immunofluorescence. Transfected cells were washed with PBS, fixed in $4 \%$ paraformaldehyde, permeabilized in $1 \%$ Triton X-100 for 5 min and blocked with 5\% bovine serum albumin in PBS containing 0.5\% Triton X-100 for $1 \mathrm{~h}$. Intact PDCD4 (aa 1-469), truncated 1 (aa 164-469) and truncated 3 (aa 327-440) were detected using anti-PDCD4 (sc-27123; Santa Cruz Biotechnology). Truncated 2 (aa 164-275) was detected using anti-PDCD4 (sc-376430; Santa Cruz Biotechnology). Cells were washed with PBS and incubated with appropriate secondary fluorophore-conjugated antibody for $1 \mathrm{~h}$ at room temperature, washed with PBS and mounted using Prolong Anti-fade (Sigma-Aldrich, Carlsbad, CA). Secondary antibody used for detection of intact PDCD4, truncated 1 and truncated 3 was Alexa Fluor ${ }^{\circledR} 488$ donkey anti-goat IgG $(\mathrm{H}+\mathrm{L})$. Alexa Fluor ${ }^{\circledR} 488$ goat anti-mouse IgG $(\mathrm{H}+\mathrm{L})$ was used to detect truncated 2. Cells were examined and captured using an Olympus CX71 fluorescence microscope (Olympus, Tokyo, Japan).

Cell viability assay. Viability of transfected cells was determined using the 3-(4,5-dimethylthiazolyl)-2,5-diphenyltetrazoliumbromide (MTT) assay (Sigma). Cells were plated in 96-well plates (1,500 cells per well) and incubated under normal culture conditions. After $24 \mathrm{~h}$, the cells were treated with $0.5 \mathrm{mg} / \mathrm{ml} \mathrm{MTT}$ for $4 \mathrm{~h}$ and lysed with dimethyl sulfoxide (DMSO). Absorbance rates were measured at 550-560 nm using a microplate reader (Bio-Rad, Hercules, CA).

Measurement of apoptotic cell death. Annexin V-FITC/ PI double staining assays were performed to detect apoptosis. Following the manufacturer's instructions (Apoptosis Detection kit, KeyGEN, Nanjing, China), cells were trypsinized, washed twice with cold PBS, then resuspended in $200 \mu \mathrm{l}$ binding buffer. Annexin V-FITC was added to a final concentration of $0.5 \mu \mathrm{g} / \mathrm{ml}$. In addition samples were incubated at room temperature in the dark. After $20 \mathrm{~min}, 300 \mu \mathrm{l}$ binding buffer containing $0.5 \mu \mathrm{g} / \mathrm{ml}$ PI was added and samples were immediately analyzed on a FACSCalibur flow cytometer (Becton-Dickinson Medical Devices, Shanghai, China). Cells in the stage of early apoptosis were defined as $\mathrm{FITC}^{+} / \mathrm{PI}^{-}$cells.

Migration assay. Migration was assessed in Boyden chambers containing polycarbonate filters with $8-\mu \mathrm{m}$ pore size (Costar, Bodenheim, Germany). The lower compartment was filled in DMEM with 10\% FBS used as a chemoattractant and the filter was placed above. Cells were harvested by trypsinization 


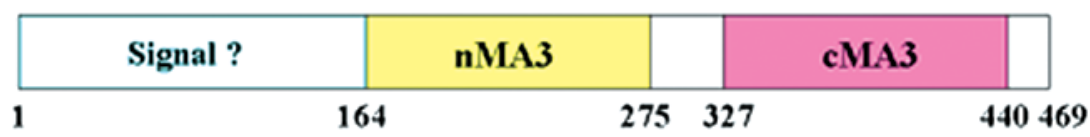

Full length

1-469aa

Truncation 1

$164-469$ aa

Truncation 2

164-275aa

Truncation 3

$327-440$ aล

Figure 1. Schematic diagram of the functional regions of PDCD4 protein. The PDCD4 is formed with mMA3 (orange) and cMA3 (magenta) domains. Nuclear localization signal was not confirmed.

Table I. Primers used to generate intact and truncated forms of PDCD4.

\begin{tabular}{ll}
\hline Region of PDCD4 amplified & \multicolumn{1}{c}{ Primers (5'-3') } \\
\hline aa 1-469 (intact) & F: AAGCTT TACCTATATCTTTTACTCGT \\
& R: GAATTC CTAGTAGCTCTCAGGTTTAA \\
aa 164-469 (truncated 1) & F: AAGCTT GTTCGTTTTCGGTTT \\
& R: GAATTC CTAGTAGCTCTCAGGTTTAA \\
aa 164-275 (truncated 2) & F: AAGCTT GTTCGTTTTCGGTTT \\
& R: GAATTC ACATAAGATTCCATC TCCAA \\
aa 327-440 (truncated 3) & F: AAGCTT ACAATTTCTCTAACTATACG \\
& R: GAATTC GGAAATTATTCCAGCCTGAA \\
\hline
\end{tabular}

Italics indicates HindIII restriction sites; underlined indicates EcoRI restriction sites. F, forward. R, reverse.

and resuspended in DMEM without FBS. Cell suspensions $(600 \mu \mathrm{l})$ at a density of $3 \times 10^{4}$ cells $/ \mathrm{ml}$ were placed in the upper compartment of the chambers. After incubation at $37^{\circ} \mathrm{C}$ for $4 \mathrm{~h}$ filters were removed, cells were fixed, stained and counted. Each condition was assayed in triplicate and assays were repeated at least twice.

Gelatin zymography. Fifty micrograms of protein was applied to $10 \%$ polyacrylamide gels with $1 \%$ gelatin incorporated as a substrate for gelatinolytic proteases. After running the gel the SDS was removed by washing twice in $2.5 \%$ Triton X-100 for $30 \mathrm{~min}$. The gels were incubated overnight in zymography development buffer containing $50 \mathrm{mM}$ Tris-HCl ( $\mathrm{pH} 7.4)$, $2 \mathrm{mM} \mathrm{NaN}_{3}$ and $5 \mathrm{mM} \mathrm{CaCl}{ }_{2}$. After development the gels were stained for $3 \mathrm{~h}$ in $45 \%$ methanol $/ 10 \%$ glacial acetic acid containing $1 \%$ (w/v). Coomassie Blue R-250 and subsequently partially destained with the same solution without dye. The gelatinolytic activity of each MMP was qualitatively evaluated as a clear band against the blue stained gelatin background.

Flow cytometry analysis. The CXCR4 or CXCR7 cell surface expression was measured and quantified using the fluorescentantibody (24). Cells were labeled with APC-anti-CXCR4 monoclonal antibody (\#555976, BD Pharmingen ${ }^{\mathrm{TM}}$, Baltimore, MD). Mouse anti-CXCR7 (AF4227, R\&D Systems China,
Shanghai, China) and PE-conjugated goat anti-mouse antibody (\#115-116-146, Jackson ImmunoResearch Laboratories, Inc., West Grove, PA) was used for the detection of CXCR7. Expression was measured by flow cytometry using a FACSCalibur machine (BD Biosciences). Receptor expression was evaluated by the mean channel fluorescence values.

Statistical analysis. Data are presented as the mean \pm standard deviation (SD). Differences between groups were analyzed using Student's t-test for continuous variables. Statistical analysis was performed using Statistical Package for the Social Sciences (SPSS, version 17.0; SPSS, Inc.) and significance was established at $\mathrm{P}<0.05$.

\section{Results}

PDCD4 expression in human melanoma specimens. The level of PDCD4 protein in cancer tissue was significantly lower than that in normal tissue $(\mathrm{P}<0.05$, Fig. $2 \mathrm{~B})$. To determine whether PDCD4 mRNA was also reduced, real-time PCR analysis was performed. Results show that the level of PDCD4 mRNA expression was coincident with the level of protein expression $(\mathrm{P}<0.05$, Fig. 2A). Furthermore, the immunohistochemical staining results showed PDCD4 proteins were located both in nucleus and cytoplasm (Fig. 3A). 

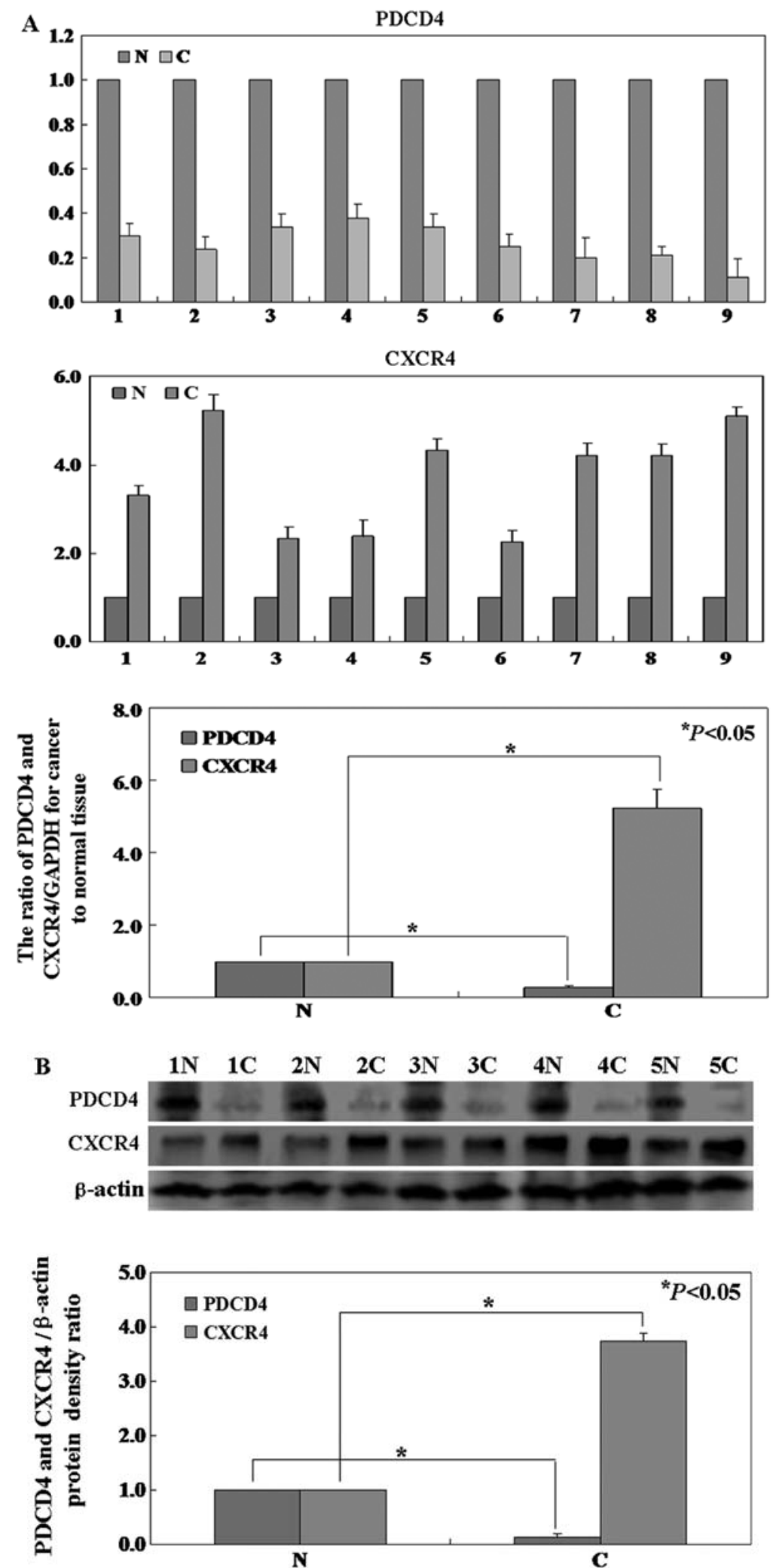

Figure 2. (A) The level of PDCD4 and $C X C R 4$ mRNA measured in specimens using real-time PCR. The level of $P D C D 4$ mRNA was lower in cancer tissues than matched normal tissues $(\mathrm{P}<0.05)$. However, the level of $C X C R 4$ mRNA was higher in cancer tissues than matched normal tissues $(\mathrm{P}<0.05)$. The $G A D P H$ was used as an internal control. (B) Representative results of two paired of gastric cancer and corresponding normal tissue by western blotting with PDCD4 and CXCR4 antibodies. PDCD4 protein expression was lower in cancer tissues than matched normal tissues $(\mathrm{P}<0.05)$. CXCR4 protein showed a higher level in cancer tissues than matched normal tissues $(\mathrm{P}<0.05)$. $\beta$-actin was used as an internal control. N, normal; $\mathrm{C}$, cancer.

Evaluation of the $m R N A$ and protein levels of different PDCD4 fragments in B16 cells. B16 cells were transfected with PDCD4 fragments that included: intact (aa 1-469), trun- cated 1 (aa 164-469), truncated 2 (aa 164-275) and truncated 3 (aa 327-440). Levels of PDCD4 fragments were detected using immunofluorescence and RT-PCR assays. In RT-PCR 

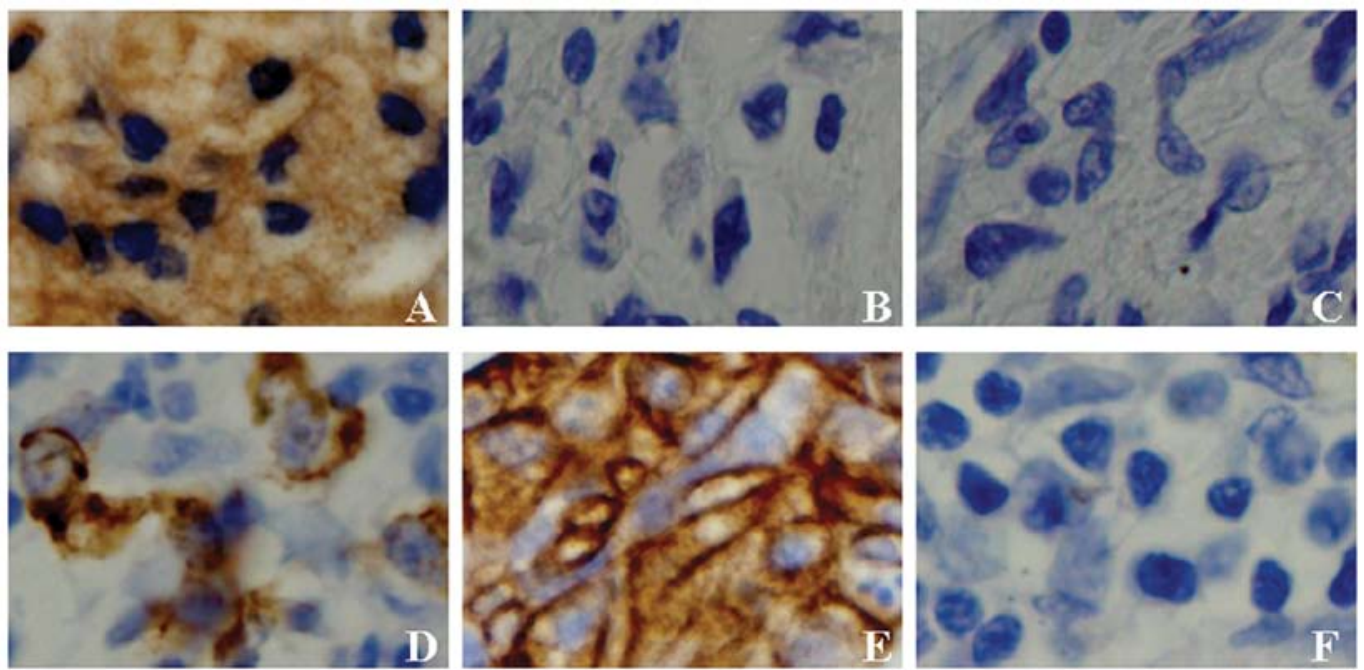

Figure 3. Immunohistochemical staining for PDCD4 and CXCR4 protein in specimens. PDCD4 was stained yellow with granules and localized to the nucleus and cytoplasm. CXCR4 was localized to the cytomembrane. The nuclei were counterstained with hematoxylin. (A and D) Paired normal tissue. (B and E) Gastric cancer tissue. (C and F) Negative control.

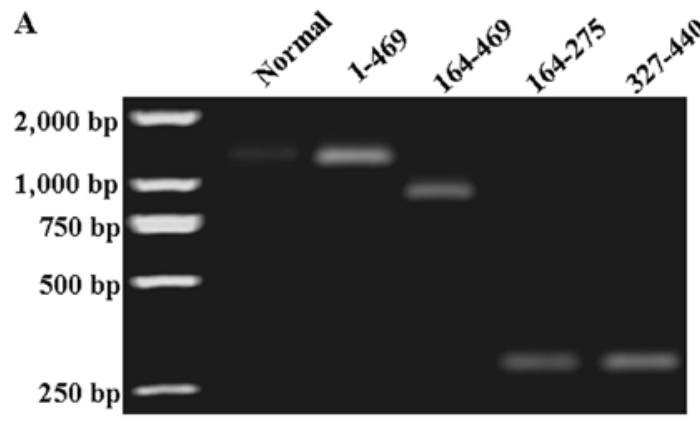

Figure 4. Detection of PDCD4 following transfection. (A) PDCD4 mRNA levels in B16 cells and transfected B16 cells detected using RT-PCR. (B) Detection of PDCD4 in transfected and untransfected B16 cells by immunofluorescence. Intact PDCD4 proteins were located predominantly in the nucleus. Truncated PDCD4 fragments were observed to localize in the cytoplasm.

B
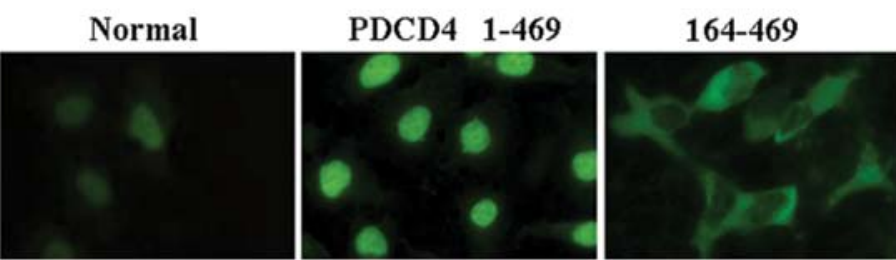

$164-275$ $327-440$
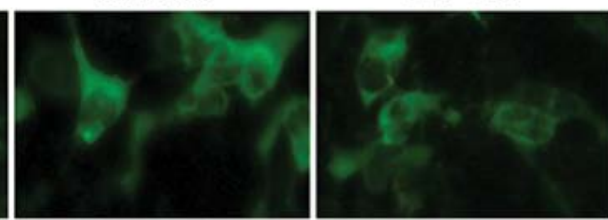

assay, levels of PDCD4 mRNA were lower in B16 cells than in treated cells (Fig. 4A). In the immunofluorescence assay, levels of PDCD4 proteins were lower in untransfected B16 cells compared with transfected B16 cells (Fig. 4B). The results showed that intact PDCD4 proteins were able to shuttle between nucleus and cytoplasm. Under normal growth conditions, intact PDCD4 proteins were located predominantly in the nucleus. However, truncated PDCD4 fragments were observed to localize in the cytoplasm (Fig. 4B).

Upregulation of PDCD4 expression in B16 cell line results in decreased proliferation, increased apoptosis and diminished migration. We then investigated the functions of PDCD4 fragments in B16 cell line. The MTT assay showed that the proliferation ratio of intact PDCD4-expressing B16 cells was decreased compared with untransfected cells $(\mathrm{P}<0.05$, Fig. 5A). Truncated 1 (aa 164-469) and truncated 3 (aa 327-440) had similar effects with intact PDCD4 on B16 cells. However, truncated 2 (aa 164-275) showed no effects on B16 cells. We determined that the percentage of apoptosis by using Annexin V and PI doublestaining in untreated B16 cells was $0.34 \%$, significantly lower compared to intact PDCD4 (2.71\%), truncated 1 (2.68\%) and truncated $3(3.25 \%)$ cells $(\mathrm{P}<0.05$, Fig. $5 \mathrm{~B})$. Truncated 2 cells $(0.41 \%)$ showed no significant changes compared with untreated ones ( $\mathrm{P}>0.05$, Fig. 5B). To quantitatively assess the cell migration rate, transwell migration assay was applied. The cells in intact PDCD4, truncated 1 and truncated 3 groups showed 54, 56 and $53 \%$ less migration, respectively, than the untreated ones $(\mathrm{P}<0.05$, Fig. $6 \mathrm{~A})$. We found that no significantly less of the truncated 2 cells migrated to the lower membrane compared to control cells ( $\mathrm{P}>0.05$, Fig. 6A). In addition, we evaluated expression of MMPs and observed the levels of MMP-2 in intact PDCD4, truncated 1 and truncated 3 cell lines were lower than that in untreated and truncated 2 cell lines (Fig. 6B). 

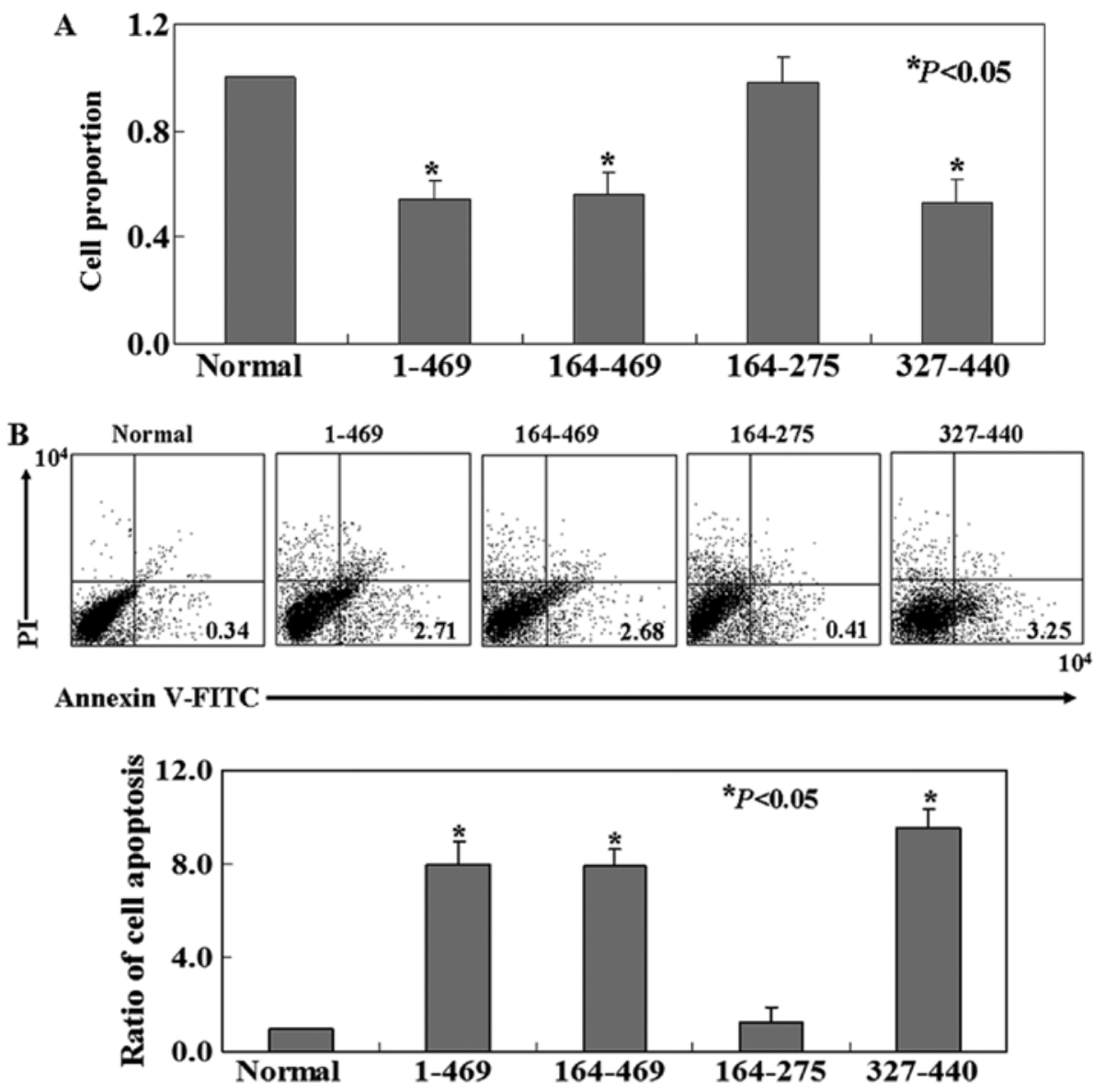

Figure 5. Expression of intact PDCD4 protein and truncated PDCD4 fragments regulates growth in B16 cells. (A) MTT assays were performed to determine the ratio of growth inhibition associated with each of the PDCD4 fragments transfected into B16 cells compared with untransfected cells. (B) Flow cytometric analysis of B16 cell apoptosis induced by PDCD4 fragments. The percentage of cell apoptosis was determined by flow cytometry with Annexin-V/FITC and PI double staining.

Cell surface expression of CXCR4 on human melanoma specimens and B16 cell lines. In order to determine the level of CXCR4 protein and mRNA in human melanoma specimens, western blotting, IHC and real-time PCR were performed respectively. The results showed both CXCR4 protein and mRNA were decreased in cancer tissues compared with normal tissues (Fig. 2). The immunohistochemical staining results showed CXCR4 proteins were located in cytomembrane (Fig. 3D and E). B16 cells with PDCD4 different fragment treatment expressed different relative levels of the cell surface receptor CXCR4. We observed that untreated B16 (55.8\%) and truncated $2(67.2 \%)$ cell lines stained highly positive for CXCR4 (Fig. 6C). In contrast, CXCR4 was expressed in intact PDCD4 (4.4\%), truncated 1 (4.2\%) and truncated $3(6.6 \%)$ cell lines at lower levels (Fig. 6C). As shown in Fig. 6C, this is not in striking difference to CXCR7 expression, which was barely expressed on the cell lines.

\section{Discussion}

PDCD4 has been characterised as a new tumor suppressor gene, which is downregulated in several human malignancies (14-18). Consistent with our results, we also found the levels of PDCD4 protein and mRNA were lower in cancer tissues than that in normal tissues. Yang et al (22) found that microRNA-21
(miR-21) regulates the metastatic behavior of B16 melanoma cells by promoting cell proliferation, survival and migration/ invasion. MicroRNA (miR) target prediction databases suggest that PDCD4 is regulated by miR-21 $(25,26)$. The expression of PDCD4 is increased during apoptosis $(27,28)$ and decreased during human and mouse carcinogenesis $(29,30)$. Overexpression of PDCD4 inhibits tumorigenesis and tumor progression in a transgenic-mouse model and inhibits tumor cell invasion in vitro (27-31). We also confirmed the antitumor activities of PDCD4 in B16 cells. These results provided the first direct evidence for an essential role of the PDCD4 in melanoma.

Furthermore, the main purpose of our study was to identify the roles of PDCD4 protein distinct domains. We designed

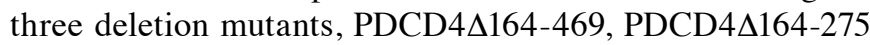
and PDCD4 $\Delta 327-440$. Residues 1-140 of human PDCD4 protein were an RNA-binding domain, residues 164-275 were nMA3 and residues 327-440 were cMA3 (9). The localization of PDCD4 has been reported to vary in a cell type-dependent manner (27). One explanation for this variability is that PDCD4 cycles between the nucleus and cytoplasm (32). However, a nuclear localization signal has not been formally identified. Interesting, we found only intact PDCD4 protein was able to shuttle between the nucleus and the cytoplasm. The results indicated that the nuclear localization signal of PDCD4 may 
A

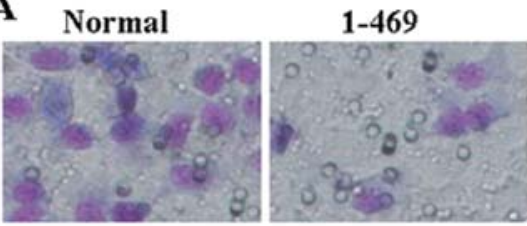

164-469

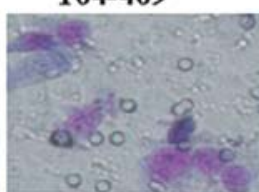

164-275

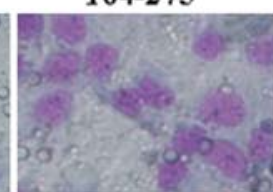

$327-440$

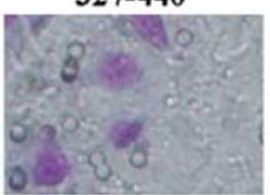

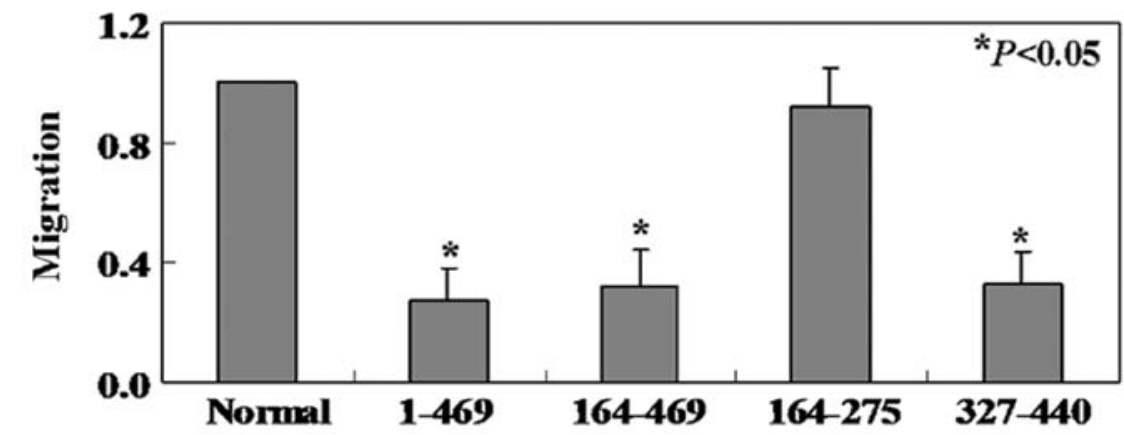

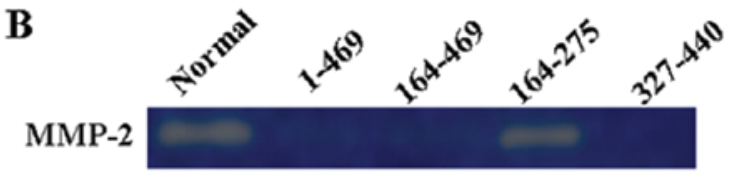

C

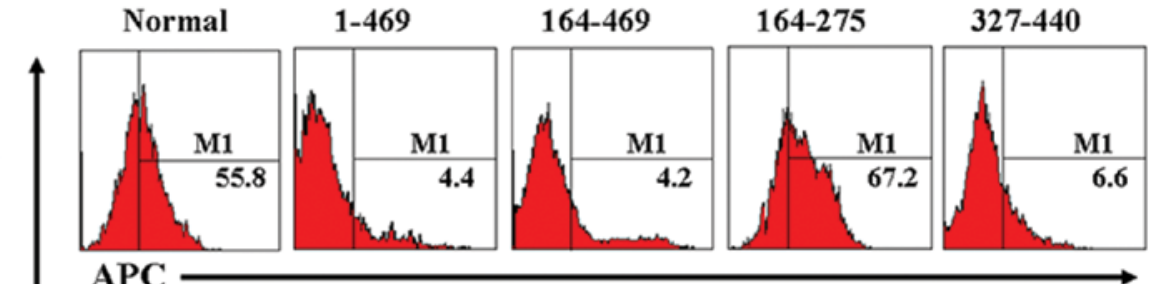

CXCR4

Figure 6. Distinct effects of different PDCD4 fragments on metastatic behavior of B16 cells. (A) Transwell assays were performed. Cells that migrated to the bottom side of the membrane were stained and counted. (B) Effect of PDCD4 fragments on gelatinolytic activities of MMP-2. Gelatinolytic activity of secreted MMP-2 of B16 cells was analyzed by zymography. (C) Expression of CXCR4 and CXCR7 on B16 cells. Flow cytometry was performed for detection of CXCR4 and CXCR7.

be included in residues 1-140. The only direct molecular function identified for PDCD4 is bound to and inhibits the function of the mammalian mRNA initiation factor eIF4A $(11,33)$. As noted in the introduction, mutational analysis and NMR binding analysis have shown that PDCD4 uses both MA3 domains to interact with eIF4A $(10,11)$. However, another study also demonstrated that the cMA3 domain alone is sufficient for the inhibition of RNA helicase and translation (13). Consistent with previous studies, our results showed that

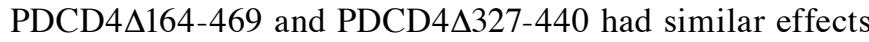
with intact PDCD4 on B16 cells. However, PDCD4 $164-275$ showed no biological activity.

Melanoma is an extremely aggressive disease with high metastatic potential (34). MMP-2 (gelatinase A) and MMP-9 (gelatinase B) are the major MMPs secreted by activated inflammatory macrophages $(35,36)$. MMP-2 is released as a latent enzyme and must be activated to degrade the matrix (37). In our study, intact PDCD4, PDCD4 164-469 and PDCD4 $327-440$ were able to reduce the gelatinolytic activity of MMP-2. Previous studies have proven that CXCR4 is a major chemokine receptor expressed in many types of cancer cells $(38,39)$. CXCR4/SDF-1 axis plays a major role in migration and adhesion of various tumor cells, including colon cancer (40), breast cancer $(41,42)$ and melanoma $(43,44)$. Consistent with previous studies, we found that CXCR4 was highly expressed in melanoma tissue compared to normal tissue by real-time PCR, western blotting and IHC. Interesting, we confirmed that CXCR4 was decreased in B16 cells after treatment with

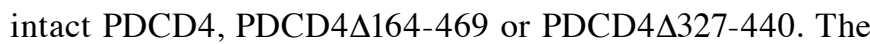
biological significance of CXCR7 receptor expression on 
B16 cells was not found. Our data presented from this study provide evidence that PDCD4 could inhibit mobility of B16 cells by regulating CXCR4 expression. However, the mechanism remains unclear.

Above all, our study demonstrated that: i) the levels of PDCD4 mRNA and protein were deficient in melanoma, however, CXCR4 showed a higher level in cancer tissues than normal tissues; ii) the tumor suppressor activity of PDCD4 and its fragments in vitro. Especially, PDCD4 inhibited mobility of B16 cells by suppressing MMP-2 activity and CXCR4 expression. This is the first report to demonstrate that different fragments of PDCD4 protein played distinct roles in B16 cells. This report may provide novel information for melanoma treatment.

\section{Acknowledgements}

We thank Dr Zhang Jin for pCDNA3.1 plasmid and Dr Yu Miao for technical assistance.

\section{References}

1. Jemal A, Bray F, Center MM, et al: Global cancer statistics. CA Cancer J Clin 61: 69-90, 2011.

2. Haass NK and Herlyn M: Normal human melanocyte homeostasis as a paradigm for understanding melanoma. J Investig Dermatol Symp Proc 10: 153-163, 2005.

3. Haass NK, Smalley KS and Herlyn M: The role of altered cellcell communication in melanoma progression. J Mol Histol 35 309-318, 2004

4. Wang Q, Sun ZX, Allgayer H, et al: Downregulation of E-cadherin is an essential event in activating beta-catenin/ Tcf-dependent transcription and expression of its target genes in Pdcd4 knockdown cells. Oncogene 29: 128-138, 2010.

5. Shibahara K, Asano M, Ishida Y, et al: Isolation of a novel mouse gene MA-3 that is induced upon programmed cell death. Gene 166: 297-301, 1995.

6. Jansen AP, Camalier CE and Colburn NH: Epidermal expression of the translation inhibitor programmed cell death 4 suppresses tumorigenesis. Cancer Res 65: 6034-6041, 2005.

7. Soejima $\mathrm{H}$, Miyoshi $\mathrm{O}$, Yoshinaga $\mathrm{H}$, et al: Assignment of the programmed cell death 4 gene (PDCD4) to human chromosome band 10q24 by in situ hybridization. Cytogenet Cell Genet 87 : 113-114, 1999.

8. Cmarik JL, Min H, Hegamyer G, et al: Differentially expressed protein PDCD4 inhibits tumor promoter-induced neoplastic transformation. Proc Natl Acad Sci USA 96: 14037-14042, 1999.

9. Chang JH, Cho YH, Sohn SY, et al: Crystal structure of the eIF4A-PDCD4 complex. Proc Natl Acad Sci USA 106: 3148-3153, 2009.

10. Suzuki C, Garces RG, Edmonds KA, et al: PDCD4 inhibits translation initiation by binding to eIF4A using both its MA3 domains. Proc Natl Acad Sci USA 105: 3274-3279, 2008.

11. Yang HS, Cho MH, Zakowicz H, et al: A novel function of the MA-3 domains in transformation and translation suppressor Pdcd4 is essential for its binding to eukaryotic translation initiation factor 4A. Mol Cell Biol 24: 3894-3906, 2004.

12. Zakowicz H, Yang HS, Stark C, et al: Mutational analysis of the DEAD-box RNA helicase eIF4AII characterizes its interaction with transformation suppressor Pdcd4 and eIF4GI. RNA 11: 261-274, 2005

13. LaRonde-LeBlanc N, Santhanam AN, Baker AR, et al: Structural basis for inhibition of translation by the tumor suppressor Pdcd 4 Mol Cell Biol 27: 147-156, 2007.

14. Chen Y, Knosel T, Kristiansen G, et al: Loss of PDCD4 expression in human lung cancer correlates with tumour progression and prognosis. J Pathol 200: 640-646, 2003.

15. Zhang H, Ozaki I, Mizuta $\mathrm{T}$, et al: Involvement of programmed cell death 4 in transforming growth factor-betal-induced apoptosis in human hepatocellular carcinoma. Oncogene 25: 6101-6112, 2006.
16. Afonja O, Juste D, Das S, et al: Induction of PDCD4 tumor suppressor gene expression by RAR agonists, antiestrogen and HER-2/neu antagonist in breast cancer cells. Evidence for a role in apoptosis. Oncogene 23: 8135-8145, 2004.

17. Mudduluru G, Medved F, Grobholz R, et al: Loss of programmed cell death 4 expression marks adenoma-carcinoma transition, correlates inversely with phosphorylated protein kinase $\mathrm{B}$, and is an independent prognostic factor in resected colorectal cancer. Cancer 110: 1697-1707, 2007.

18. Motoyama K, Inoue H, Mimori K, et al: Clinicopathological and prognostic significance of PDCD4 and microRNA-21 in human gastric cancer. Int J Oncol 36: 1089-1095, 2010.

19. Jin H, Kim TH, Hwang SK, et al: Aerosol delivery of urocanic acid-modified chitosan/programmed cell death 4 complex regulated apoptosis, cell cycle, and angiogenesis in lungs of K-ras null mice. Mol Cancer Ther 5: 1041-1049, 2006.

20. Jansen AP, Camalier CE, Stark C, et al: Characterization of programmed cell death 4 in multiple human cancers reveals a novel enhancer of drug sensitivity. Mol Cancer Ther 3: 103-110, 2004.

21. Caporali S, Alvino E, Levati L, et al: Down-regulation of the PTTG1 proto-oncogene contributes to the melanoma suppressive effects of the cyclin-dependent kinase inhibitor PHA-848125. Biochem Pharmacol 84: 598-611, 2012.

22. Yang CH, Yue J, Pfeffer SR, et al: MicroRNA miR-21 regulates the metastatic behavior of B16 melanoma cells. J Biol Chem 286: 39172-39178, 2011.

23. Rothhammer T, Hahne JC, Florin A, et al: The Ets-1 transcription factor is involved in the development and invasion of malignant melanoma. Cell Mol Life Sci 61: 118-128, 2004.

24. Amara A, Gall SL, Schwartz O, et al: HIV coreceptor downregulation as antiviral principle: SDF-1alpha-dependent internalization of the chemokine receptor CXCR4 contributes to inhibition of HIV replication. J Exp Med 186: 139-146, 1997.

25. Griffiths-Jones S, Grocock RJ, van Dongen S, et al: miRBase: microRNA sequences, targets and gene nomenclature. Nucleic Acids Res 34: D140-D144, 2006.

26. Betel D, Wilson M, Gabow A, et al: The microRNA.org resource: targets and expression. Nucleic Acids Res 36: D149-D153, 2008.

27. Lankat-Buttgereit B and Goke R: The tumour suppressor Pdcd4: Recent advances in the elucidation of function and regulation. Biol Cell 101: 309-317, 2009.

28. Allgayer H: Pdcd4, a colon cancer prognostic that is regulated by a microRNA. Crit Rev Oncol Hematol 73: 185-191, 2010.

29. Yang HS, Jansen AP, Nair R, et al: A novel transformation suppressor, Pdcd4, inhibits AP-1 transactivation but not NF- $\mathrm{BB}$ or ODC transactivation. Oncogene 20: 669-676, 2001.

30. Schmid T, Jansen AP, Baker AR, et al: Translation inhibitor Pdcd4 is targeted for degradation during tumor promotion. Cancer Res 68: 1254-1260, 2008.

31. Leupold JH, Yang HS, Colburn NH, et al: Tumor suppressor Pdcd4 inhibits invasion/intravasation and regulates urokinase receptor (u-PAR) gene expression via $\mathrm{Sp}$-transcription factors. Oncogene 26: 4550-4562, 2007.

32. Böhm M, Sawicka K, Siebrasse JP, et al: The transformation suppressor protein Pdcd4 shuttles between nucleus and cytoplasm and binds RNA. Oncogene 22: 4905-4910, 2003.

33. Yang HS, Jansen AP, Komar AA, et al: The transformation suppressor Pdcd4 is a novel eukaryotic translation initiation factor 4A binding protein that inhibits translation. Mol Cell Biol 23: 26-37, 2003.

34. Tawbi HA and Kirkwood JM: Management of metastatic melanoma. Semin Oncol 34: 532-545, 2007.

35. Gibbs DF, Warner RL, Weiss SJ, et al: Characterization of matrix metalloproteinases produced by rat alveolar macrophages. Am J Respir Cell Mol Biol 20: 1136-1144, 1999.

36. Shah PK, Falk E, Badimon JJ, et al: Human monocyte-derived macrophages induce collagen breakdown in fibrous caps of atherosclerotic plaques. Potential role of matrix-degrading metalloproteinases and implications for plaque rupture. Circulation 92: 1565-1569, 1995.

37. Jackson C, Nguyen M, Arkell J, et al: Selective matrix metalloproteinase (MMP) inhibition in rheumatoid arthritis-targetting gelatinase A activation. Inflamm Res 50: 183-186, 2001.

38. Balkwill F: The significance of cancer cell expression of the chemokine receptor CXCR4. Semin Cancer Biol 14: 171-179, 2004. 
39. Kucia M, Jankowski K, Reca R, et al: CXCR4-SDF-1 signalling, locomotion, chemotaxis and adhesion. J Mol Histol 35: 233-245, 2004.

40. Schimanski CC, Schwald S, Simiantonaki N, et al: Effect of chemokine receptors CXCR4 and CCR7 on the metastatic behavior of human colorectal cancer. Clin Cancer Res 11: 1743-1750, 2005.

41. Harvey JR, Mellor P, Eldaly H, et al: Inhibition of CXCR4 mediated breast cancer metastasis: a potential role for heparinoids? Clin Cancer Res 13: 1562-1570, 2007.

42. Zhou W, Jiang Z, Liu N, et al: Down-regulation of CXCL12 mRNA expression by promoter hypermethylation and its association with metastatic progression in human breast carcinomas. J Cancer Res Clin Oncol 135: 91-102, 2009.
43. Turner RR, Ye X, Bilchik AJ, et al: Chemokine receptor CXCR4 expression in patients with melanoma and colorectal cancer liver metastases and the association with disease outcome. Ann Surg 244: 113-120, 2006.

44. Scala S, Giuliano P, Ascierto PA, et al: Human melanoma metastases express functional CXCR4. Clin Cancer Res 12: 2427-2433, 2006. 